\title{
Dissipativity and LQ Optimal Control of Linear Implicit Systems ${ }^{\dagger}$
}

\author{
Kiyotsugu TAKABA*
}

\begin{abstract}
This paper considers the linear quadratic optimal control problem for linear implicit systems based on the dissipation inequality. We derive a necessary and sufficient condition for the dissipativity with respect to a quadratic supply rate in terms of a linear matrix inequality (LMI) condition with an equality constraint. Based on this constrained LMI condition, the optimal control law is given by an implicit algebraic constraint among system variables. We also show that the present constrained LMI condition easily reduces to an LMI without any equality constraints.
\end{abstract}

Key Words: linear implicit system, dissipativity, LQ optimal control, constrained linear matrix inequality

\section{Introduction}

This paper is concerned with the linear quadratic (LQ) optimal control problem for a class of dynamical systems called the linear implicit systems based on the dissipation inequality.

The linear implicit system is defined as a system described by a set of first-order linear implicit differential/algebraic equations ${ }^{1)}$. Although the linear implicit system representation is very simple, it has great flexibility in modeling of physical systems. For this reason, the basic theory of the linear implicit system has been extensively studied in the literature (see 1), 4), 5), 9) 11) and the references therein).

It is well known that the theory of dissipative systems plays an important role in the optimal control problems. Willems ${ }^{15)}$ considered the dissipation inequality and LQ optimal control of a linear state-space system using linear matrix inequalities (LMIs) and algebraic Riccati equations (AREs). Geerts ${ }^{2)}$ and Kawamoto and Katayama ${ }^{8)}$ applied the dissipation inequality approach to the LQ optimal control for a descriptor system. For linear implicit systems with consistent initial conditions, van der Geest and Rantzer ${ }^{6)}$ derived an LMI condition for dissipativity with respect to a quadratic supply rate, and characterized the optimal control law in terms of the solution of this LMI. It may be also noted that the dissipation inequality of a system described by high-order implicit differential/algebraic equations was considered by using

\footnotetext{
$\dagger$ 38th IEEE Conf. Decision and Contr. (1999 · 12)

* Graduate School of Informatics, Kyoto University, YoshidaHonmachi, Sakyo-ku, Kyoto

(Received October 17, 2000)

(Revised March 7, 2001)
}

the quadratic differential form in the behavioral framework ${ }^{13), 17)}$.

In this paper, we consider the dissipativity of a linear implicit system in connection with the dissipation theory for state-space systems ${ }^{15)}$. A necessary and sufficient condition for dissipativity of a linear implicit system with respect to a quadratic supply rate is derived in terms of an LMI condition with an equality constraint. It turns out that the present result is a generalization of the previous result for descriptor systems ${ }^{8)}$. By using the solution to the constrained LMI, we also characterize the optimal control for the LQ control problem by a certain algebraic constraint among system variables. Finally, we will show that we have no numerical difficulties in solving the constrained LMI by deriving an equivalent LMI condition.

\section{Linear Implicit System}

We consider a linear implicit system $\Sigma$ described by

$$
E \dot{\xi}(t)=F \xi(t)
$$

where $F, E \in \mathbf{R}^{m \times n}, r:=\operatorname{rank} E \leq \min \{m, n\}$, and $\xi(t) \in \mathbf{R}^{n}$ is the system variable vector consisting of the inputs, outputs, states and the other redundant variables. It should also be noted that, unlike the conventional state-space framework, we do not make a priori distinction among inputs, outputs and states in the vector $\xi(t)$. Moreover, since the pair $(E, F)$ uniquely defines the dynamical system $\Sigma$, we write the implicit system as $\Sigma(E, F)$.

In the behavioral setting ${ }^{16)}$, a dynamical system is characterized in terms of the behavior $\mathcal{B}$, the set of all possible trajectories of the system variables. We can define the behavior of $\Sigma(E, F)$ as the set of solutions to the differential/algebraic equation (1). 
As well-known, an implicit system may admit impulsive response if its initial condition, denoted by $\xi_{0}$, is not consistent with algebraic constraints in (1). It is helpful to introduce the impulsive-smooth distributions $\mathcal{C}_{\text {imp }}$ in order to describe the impulsive behavior ${ }^{2) ~ 5), 7)}$. Thus, we define two behaviors with support on $[0, \infty)$ as

$$
\begin{array}{r}
\mathcal{B}_{\mathrm{imp}}\left(\xi_{0}\right)=\left\{\xi \in \mathcal{C}_{\mathrm{imp}} \mid E \dot{\xi}(t)=F \xi(t) \quad \forall t>0,\right. \\
\left.\xi(0-)=\xi_{0}\right\} \\
\mathcal{B}_{\mathrm{sm}}\left(\xi_{0}\right)=\mathcal{B}_{\mathrm{imp}}\left(\xi_{0}\right) \cap \mathcal{C}_{\mathrm{sm}}
\end{array}
$$

where $\mathcal{C}_{\text {imp }}$ and $\mathcal{C}_{\text {sm }}$ are defined in Appendix A. We also define the following sets of initial conditions.

$$
\begin{aligned}
& \Xi_{\mathrm{imp}}=\left\{\xi_{0} \in \mathbf{R}^{n} \mid \mathcal{B}_{\mathrm{imp}}\left(\xi_{0}\right) \neq \emptyset\right\} \\
& \Xi_{\mathrm{sm}}=\left\{\xi_{0} \in \mathbf{R}^{n} \mid \mathcal{B}_{\mathrm{sm}}\left(\xi_{0}\right) \neq \emptyset\right\} \subseteq \Xi_{\mathrm{imp}}
\end{aligned}
$$

The system representation (1) is simple but very general as shown in the following examples.

Example 1. The descriptor form representation

$$
E_{d} \dot{x}(t)=A_{d} x(t)+B_{d} u(t)
$$

is equivalent to (1) with

$$
E=\left[\begin{array}{ll}
E_{d} & 0
\end{array}\right], \quad F=\left[\begin{array}{ll}
A_{d} & B_{d}
\end{array}\right], \quad \xi(t)=\left[\begin{array}{l}
x(t) \\
u(t)
\end{array}\right]
$$

Example 2. We consider a system described by the so-called kernel representation

$$
R_{0} w(t)+R_{1} \frac{d w(t)}{d t}+\cdots+R_{p} \frac{d^{p} w(t)}{d t^{p}}=0
$$

This equation is equivalent to (1) with

$$
\begin{gathered}
\xi(t)=\left[\begin{array}{c}
w(t) \\
\frac{d w(t)}{d t} \\
\vdots \\
\frac{d^{p} w(t)}{d t^{p}}
\end{array}\right], E=\left[\begin{array}{cccc}
0 & 0 & \cdots & 0 \\
I & 0 & \cdots & 0 \\
& \ddots & \ddots & \vdots \\
0 & & I & 0
\end{array}\right] \\
F=\left[\begin{array}{cccc}
R_{0} & R_{1} & \cdots & R_{p} \\
0 & I & & 0 \\
\vdots & \ddots & \ddots & \\
0 & \cdots & 0 & I
\end{array}\right]
\end{gathered}
$$

Remark. The system representation (1) is a firstorder version of the kernel representation with $R_{0}=F$, $R_{1}=-E$ and $p=1$. Thus the system representation (1) inherits the properties of the kernel representation due to Willems ${ }^{16)}$.

An advantage of the first-order implicit system representation is that we can translate some properties of a dynamical system into the conditions of the non-square matrix pencil $F-s E$ as shown below.
Definition 1. The system representation (1), or simply the pair $(E, F)$, is said to be minimal if

$$
\max _{s \in \mathbf{C}} \operatorname{rank}(F-s E)=m
$$

There are several different definitions of the minimality of a linear implicit systems $\left.{ }^{1}{ }^{1}, 9\right), 16$ ). Among them, the condition in Definition 1 is equivalent to the minimality of the kernel reparesentation in the behavioral theory ${ }^{16)}$. Since the equation (1) represents $m$ constraints described by linear differential/algebraic equations, the minimality in Definition 1 implies that there are no redundant constraints in (1).

Moreover, $\Xi_{\mathrm{imp}}=\mathbf{R}^{n}$ holds if $(E, F)$ is minimal ${ }^{5)}$. That is, there always exists a $\xi \in \mathcal{B}_{\text {imp }}\left(\xi_{0}\right)$ for any initial condition $\xi_{0} \in \mathbf{R}^{n}$.

Definition 2. The system $\Sigma(E, F)$ is said to be controllable in the behavioral sense if for any $\xi_{1}, \xi_{2} \in \mathcal{B}$ there exists a time $T \geq 0$ and a $\xi \in \mathcal{B}$ satisfying

$$
\xi(t)=\left\{\begin{array}{cc}
\xi_{1}(t), & t<0 \\
\xi_{2}(t-T), & t>T
\end{array}\right.
$$

where $\mathcal{B}$ is the smooth behavior defined on $\mathbf{R}$;

$$
\mathcal{B}=\left\{\xi \in \mathcal{C}^{\infty} \mid E \dot{\xi}(t)=F \xi(t), \forall t \in \mathbf{R}\right\}
$$

A necessary and sufficient condition for $\Sigma(E F)$ to be controllable in the behavioral sense is that $\operatorname{rank}(F-s E)$ is constant for any $s \in \mathbf{C}^{6)}$, 16)

Definition 3. The implicit system $\Sigma(E, F)$ is said to be impulse controllable if there exists a trajectory $\xi \in \mathcal{C}_{\text {sm }}$ for any initial condition $\xi_{0}$ such that $\mathcal{B}_{\text {imp }}\left(\xi_{0}\right) \neq \emptyset$. In other words, the impulse controllability implies that $\Xi_{\mathrm{imp}}=\Xi_{\mathrm{sm}}$.

Lemma 1. We assume that $(E, F)$ is minimal. Then, $\Sigma(E, F)$ is impulse controllable if and only if

$$
\operatorname{im} E+F \operatorname{ker} E=\mathbf{R}^{m}
$$

In this case, we have $\Xi_{\mathrm{sm}}=\mathbf{R}^{n}$.

\section{Dissipation Theory}

We define the dissipativity of the linear implicit system $\Sigma(E, F)$ as follows.

Definition 4. The implicit system $\Sigma(E, F)$ is said to be dissipative with respect to $s(\xi)$ if there exist a function $v(E \xi)$ satisfying $v(0)=0$ and the dissipation inequality

$$
\begin{aligned}
\int_{0-}^{\tau} s(\xi(t)) d t+v(E \xi(\tau)) \geq v\left(E \xi_{0}\right), \\
\forall \tau \geq 0, \forall \xi \in \mathcal{B}_{\mathrm{sm}}\left(\xi_{0}\right), \forall \xi_{0} \in \Xi_{\mathrm{sm}}
\end{aligned}
$$

The functions $s(\xi)$ and $-v(E \xi)$ are called the supply rate and storage function, respectively. 
Remark. The vector $E \xi(t)$ represents the dynamic part of $\xi(t)$, and is equivalent to the state vector of the state-space model. Moreover, since the components of $\xi(t)$ belonging to ker $E$ represent the static constraints, they do not affect on the energy stored in the system. Therefore, without loss of generality, we can assume that $v$ is the function of $E \xi(t)$. It may also be noted that, in the behavioral context, Trentelman and Willems ${ }^{13)}$ showed that every storage function is a function of state variables.

We henceforth make the following assumptions.

(A1) The system $\Sigma(E, F)$ is controllable in the behavioral sense.

(A2) The pair $(E, F)$ is minimal.

(A3) The system $\Sigma(E, F)$ is impulse controllable.

Remark. Van der.Geest and Rantzer ${ }^{6}$ ) derived an LMI condition for dissipativity of $\Sigma(E, F)$ by restricting the initial condition $\xi_{0}$ to be consistent, i.e. $\xi(0+)=\xi_{0}$. On the contrary, we will derive a necessary and sufficient condition for dissipativity without any restrictions on $\xi_{0}$. Namely, we allow the trajectory $\xi(t)$ to have a jump at $t=0$ under the assumption (A3).

It is easy to verify that, under the assumptions (A2) and (A3), there exist nonsingular matrices $U$ and $V$ such that

$$
U E V=\left[\begin{array}{lll}
I & 0 & 0 \\
0 & 0 & 0
\end{array}\right], U F V=\left[\begin{array}{ccc}
A_{0} & B_{0} & B \\
C_{0} & I & 0
\end{array}\right]
$$

Then, by defining

$$
V^{-1} \xi(t)=\left[\begin{array}{ll}
x^{T}(t) & f^{T}(t) u^{T}(t)
\end{array}\right]^{T}
$$

we obtain

$$
\begin{aligned}
\dot{x}(t) & =A_{0} x(t)+B_{0} f(t)+B u(t) \\
f(t) & =-C_{0} x(t)
\end{aligned}
$$

Hence, the system representation (1) is equivalent to the state-space representation

$$
\dot{x}(t)=A x(t)+B u(t), \quad A:=A_{0}-B_{0} C_{0}
$$

It is obvious from the above equation that the state trajectory $x(t)$ is uniquely determined for $u(t)$ :

$$
x(t)=e^{A t} x(0-)+\int_{0-}^{t} e^{A(t-\tau)} B u(\tau) d \tau
$$

Hence, we can regard $u(t)$ as a fictitious input to the system though it may not be the actual input in general.

This transformation of representations from (1) to (11) preserves the controllability.

Lemma 2. Under the assumptions (A2)-(A3), the implicit system $\Sigma(E, F)$ is controllable in the behavioral sense if and only if the state-space system (11) is controllable.
Proof. The lemma is obvious from

$$
\begin{aligned}
\operatorname{rank}(F-s E) & =\operatorname{rank}\left\{U(F-s E) V\left[\begin{array}{ccc}
I & 0 & 0 \\
-C_{0} & 0 & I \\
0 & I & 0
\end{array}\right]\right\} \\
& =\operatorname{rank}\left[\begin{array}{ccc}
A-s I & B & B_{0} \\
0 & 0 & I
\end{array}\right]
\end{aligned}
$$

The supply rate $s(\xi)$ and the function $v(E \xi)$ are respectively the functions of $(x, u)$ and $x$, since we obtain from (8) and (10)

$$
\begin{gathered}
\xi(t)=V\left[\begin{array}{cc}
I & 0 \\
-C_{0} & 0 \\
0 & I
\end{array}\right]\left[\begin{array}{l}
x(t) \\
u(t)
\end{array}\right] \\
E \xi(t)=U^{-1}\left[\begin{array}{c}
x(t) \\
0
\end{array}\right]
\end{gathered}
$$

Therefore, the dissipativity of the implicit system $\Sigma(E, F)$ is equivalent to that of the state-space system (11) under the assumptions (A1)-(A3) (see Appendix B for the definition of dissipativity of a state-space system). We obtain the following theorem by directly applying Lemma B.1 in Appendix B to (11).

Theorem 1. Under the assumptions $(A 1)-(A 3)$, the following statements are equivalent.

(i) $\Sigma(E, F)$ is dissipative with respect to $s(\xi)$.

(ii) $v^{+}\left(E \xi_{0}\right)>-\infty, \quad \forall \xi_{0} \in \mathbf{R}^{n}$

(iii) $\int_{0-}^{\tau} s(\xi(t)) d t \geq 0$ holds for all $\tau \geq 0$ and for all $\xi \in \mathcal{B}_{\mathrm{sm}}\left(\xi_{0}\right)$ satisfying $E \xi\left(0_{-}\right)=E \xi(\tau)=0$.

Here, $v^{+}$is defined by

$$
v^{+}\left(E \xi_{0}\right)=\inf _{\xi \in \mathcal{B}_{\mathrm{sm}}\left(\xi_{0}\right)}\left\{\int_{0-}^{\infty} s(\xi(t)) d t \mid \lim _{t \rightarrow \infty} \xi(t)=0\right\}
$$

Moreover, if the system is dissipative, then the following inequality holds for any function $v(E \xi)$ satisfying (6).

$$
v(E \xi) \leq v^{+}(E \xi), \quad \forall \xi \in \mathbf{R}^{n}
$$

In the remainder of this paper, we will consider the dissipativity with respect to the quadratic supply rate

$$
s(\xi)=\xi^{T} M \xi, \quad M=M^{T} \in \mathbf{R}^{n \times n}
$$

It should be noted that there is no assumption on the sign definiteness of the weighting matrix $M$. Since $\xi$ is the linear transform of $(x, u)$, the supply rate $s(\xi)=\xi^{T} M \xi$ can be expressed as a quadratic form of $x$ and $u$. Similarly, the quadratic storage function $v$ of $E \xi$ is a quadratic function of $x$, and vice versa. Therefore, the dissipativity of the system $\Sigma(E, F)$ with respect to the quadratic supply rate $s(\xi)=\xi^{T} M \xi$ is characterized in terms of a certain matrix inequality condition. 
Theorem 2.

(i) Under the assumptions (A1)-(A3), a necessary and sufficient condition for the system $\Sigma(E, F)$ to be dissipative with respect to $s(\xi)=\xi^{T} M \xi$ is that there exists a constant matrix $X$ satisfying the constrained linear matrix inequality (CLMI)

$$
\begin{gathered}
E^{T} X=X^{T} E \\
L(X):=F^{T} X+X^{T} F+M \geq 0
\end{gathered}
$$

(ii) If the CLMI (15) has a solution $X$, then $v(E \xi)=$ $\xi^{T} E^{T} X \xi$ satisfies the dissipation inequality (6).

(iii) Let $\mathcal{X}$ denote the set of all solutions to (15). Then, there exists $X^{+} \in \mathcal{X}$ such that

$$
E^{T} X \leq E^{T} X^{+}, \quad \forall X \in \mathcal{X}
$$

Moreover, we have

$$
v^{+}(E \xi)=\xi^{T} E^{T} X^{+} \xi
$$

Proof. (i) We easily see from (13) that the supply rate $s(\xi)=\xi^{T} M \xi$ is expressed by

$$
s(\xi)=s(x, u):=\left[x^{T} \cdot u^{T}\right]\left[\begin{array}{cc}
Q & S \\
S^{T} & R
\end{array}\right]\left[\begin{array}{l}
x \\
u
\end{array}\right]
$$

where

$$
\left[\begin{array}{cc}
Q & S \\
S^{T} & R
\end{array}\right]=\left[\begin{array}{cc}
I & 0 \\
-C_{0} & 0 \\
0 & I
\end{array}\right]^{T} V^{T} M V\left[\begin{array}{cc}
I & 0 \\
-C_{0} & 0 \\
0 & I
\end{array}\right]
$$

By Lemma B.2, the state-space system (11) is dissipative with respect to the quadratic supply rate $s(x, u)$ if and only if the LMI

$$
\left[\begin{array}{cc}
A^{T} P+P A & P B \\
B^{T} P & 0
\end{array}\right]+\left[\begin{array}{cc}
Q & S \\
S^{T} & R
\end{array}\right] \geq 0
$$

has a symmetric solution $P$.

Since the dissipativity of $\Sigma(E, F)$ is equivalent to that of the state-space system (11), it suffices to prove that the LMI condition (18) is equivalent to (15).

We here define

$$
U^{-T} X V=\left[\begin{array}{ccc}
X_{1} & X_{2} & X_{3} \\
X_{4} & X_{5} & X_{6}
\end{array}\right]
$$

It follows from (7),(15a) and (19) that

$$
X_{1}=X_{1}^{T}, \quad X_{2}=0, \quad X_{3}=0
$$

Moreover, we see from (7),(15b) and (19) that

$$
\begin{gathered}
{\left[\begin{array}{ccc}
\left(\begin{array}{c}
A_{0}^{T} X_{1}+X_{1} A_{0} \\
+C_{0}^{T} X_{4}+X_{4}^{T} C_{0}
\end{array}\right) & \star & \star \\
B_{0}^{T} X_{1}+X_{4}+X_{5}^{T} C_{0} & X_{5}^{T}+X_{5} & \star \\
B^{T} X_{1}+X_{6}^{T} C_{0} & X_{6}^{T} & 0
\end{array}\right]} \\
+\left[\begin{array}{ccc}
M_{11} & \star & \star \\
M_{12}^{T} & M_{22} & \star \\
M_{13}^{T} & M_{23}^{T} & M_{33}
\end{array}\right] \geq 0
\end{gathered}
$$

where $\star$ denotes irrelevant off-diagonal blocks in a symmetric matrix, and we have partitioned $V^{T} M V$ as

$$
V^{T} M V=\left[\begin{array}{lll}
M_{11} & M_{12} & M_{13} \\
M_{12}^{T} & M_{22} & M_{23} \\
M_{13}^{T} & M_{23}^{T} & M_{33}
\end{array}\right]
$$

Post-multiplying (20) by $\left[\begin{array}{cc:c}I & 0 & 0 \\ -C_{0} & 0 & I \\ 0 & I & 0\end{array}\right]$ and premultiplying by its transpose, the CLMI (15) is reduced to the LMI

$$
\begin{aligned}
& {\left[\begin{array}{ccc}
A^{T} X_{1}+X_{1} A & X_{1} B & \star \\
\hdashline-B^{T} X_{1} & 0 & \star \\
\hdashline X_{4}-X_{5} C_{0}+\bar{B}_{0}^{T} X_{1} & X_{6} & X_{5}+X_{5}^{T}
\end{array}\right]} \\
& +\left[\begin{array}{ccc}
Q & S & \star \\
S^{T} & R & \star \\
\hdashline M_{12}^{T}-M_{22} C_{0} & M_{23}, & M_{22}
\end{array}\right] \geq 0 \quad(21)
\end{aligned}
$$

in which $X_{1}=X_{1}^{T}, X_{4}, X_{5}$ and $X_{6}$ are the unknown variables.

We assume that the CLMI (15) has a solution, namely there exist $X_{1}, X_{4}, X_{5}$ and $X_{6}$ satisfying (21). Then, we see from the upper left $2 \times 2$ block of (21) that

$$
\left[\begin{array}{cc}
A^{T} X_{1}+X_{1} A & X_{1} B \\
B^{T} X_{1} & 0
\end{array}\right]+\left[\begin{array}{cc}
Q & S \\
S^{T} & R
\end{array}\right] \geq 0
$$

Obviously, $X_{1}$ is a symmetric solution to the LMI (18).

Conversely, suppose that (18) has a solution $P$. Then, the LMI (21), or equivalently (15), is satisfied by

$$
\begin{aligned}
& X_{1}=P \\
& X_{4}=X_{5} C_{0}-B_{0}^{T} P-M_{12}^{T}+M_{22} C_{0} \\
& X_{5}=N-\frac{1}{2} M_{22}, \quad N+N^{T} \geq 0 \\
& X_{6}=-M_{23}
\end{aligned}
$$

Consequently, we have shown that $\Sigma(E, F)$ is dissipative with respect to $s(\xi)=\xi^{T} M \xi$ if and only if the CLMI (15) has a solution.

(ii) It is easily seen from the proof of (i) that the solution $X$ to (15) has the form of

$$
X=U^{T}\left[\begin{array}{ccc}
P & 0 & 0 \\
X_{4} & X_{5} & X_{6}
\end{array}\right] V^{-1}
$$

where $P$ is a solution of (18), and $X_{4}, X_{5}$ and $X_{6}$ are given by (23)-(25). We obtain from (7) and (26)

$$
E^{T} X=V^{-T}\left[\begin{array}{ccc}
P & 0 & 0 \\
0 & 0 & 0 \\
0 & 0 & 0
\end{array}\right] V^{-1}
$$

It thus follows from (13) that

$$
\xi^{T} E^{T} X \xi=\xi^{T} V^{-T}\left[\begin{array}{ccc}
P & 0 & 0 \\
0 & 0 & 0 \\
0 & 0 & 0
\end{array}\right] V^{-1} \xi=x^{T} P x
$$


Since $v=x^{T} P x$ satisfies (B. 2) by Lemma B.2, the above equation implies that $v=\xi^{T} E^{T} X \xi$ satisfies the dissipation inequality (6).

(iii) Let $\mathcal{P}$ be the set of all solutions to the LMI (18). By Lemma B.2 in Appendix B, there exists a $P^{+} \in \mathcal{P}$ such that $P \leq P^{+}$for all $P \in \mathcal{P}$. We define $X^{+}$by replacing $P$ in (26) with $P^{+}$. Then, the inequality (16) immediately follows from (27) and $P \leq P^{+}$.

Remark. The CLMI (15) is the same type of inequality as the one proposed by Masubuchi and Shimemura ${ }^{10)}$. Similar kind of matrix inequality and equation can be also found in the context of descriptor systems ${ }^{8), 12)}$. Thus, we can say that the present CLMI condition for dissipativity is a generalization of the previous result for descriptor systems $^{8)}$.

\section{Linear Quadratic Optimal Control}

In this section, we derive a formula of the optimal control law for the LQ optimal control problem with positive semi-definite weighting matrix $M$.

The LQ optimal control problem is defined as the task of finding a control law achieving

$$
J^{*}=\min _{\xi \in \mathcal{B}_{\sin }\left(\xi_{0}\right)}\left\{J \mid \lim _{t \rightarrow \infty} \xi(t)=0\right\}
$$

for the linear implicit system $\Sigma(E, F)$ and for a given quadratic performance index

$$
J=\int_{0-}^{\infty} \xi^{T}(\tau) M \xi(\tau) d \tau, \quad M=G^{T} G \geq 0
$$

We assume the following conditions as well as (A1)(A3).

(A4) (A4) $\operatorname{rank}\left[\begin{array}{c}F-j \omega E \\ G\end{array}\right]=n, \quad \forall \omega \in \mathbf{R}$

(A5) $\operatorname{ker} E \cap F^{-1} \operatorname{im} E \cap \operatorname{ker} G=\{0\}$,

where $F^{-1} \mathcal{X}:=\{x \mid F x \in \mathcal{X}\}$.

Clearly, if the minimum in (28) exists, then we have $J^{*}=v^{+}\left(E \xi_{0}\right)$, where $v^{+}$is defined in Theorem 1 with $s(\xi)=\xi^{T} M \xi$. We will show that a control law achieving the minimum in (28) does exist under the assumptions (A1)-(A5).

Theorem 3. Under the assumptions (A1)-(A5), we have

$$
\operatorname{rank} L\left(X^{+}\right)=n-m
$$

Moreover, the optimal control minimizing $J$ is given by

$$
H \xi(t)=0
$$

where $H \in \mathbf{R}^{(n-m) \times n}$ is a constant matrix satisfying $L\left(X^{+}\right)=H^{T} H$.
Proof. We again introduce the decomposition of (7) and (8). We define

$$
\left[\begin{array}{lll}
G_{1} & G_{2} & G_{3}
\end{array}\right]=G V
$$

Then, by (13) and (32), the performance index $J$ in (29) is rewritten as

$$
J=\int_{0-}^{\infty}\left[\begin{array}{l}
x(t) \\
u(t)
\end{array}\right]^{T}\left[\begin{array}{cc}
Q & S \\
S^{T} & R
\end{array}\right]\left[\begin{array}{l}
x(t) \\
u(t)
\end{array}\right] d t
$$

where

$$
\begin{aligned}
& {\left[\begin{array}{cc}
Q & S \\
S^{T} & R
\end{array}\right]=\left[\begin{array}{cc}
C^{T} C & C^{T} D \\
D^{T} C & D^{T} D
\end{array}\right]} \\
& C=G_{1}-G_{2} C_{0}, \quad D=G_{3}
\end{aligned}
$$

Therefore, the LQ optimal control problem for the implicit system $\Sigma=(E, F)$ reduces to the $\mathrm{LQ}$ regulator problem for the state-space system (11).

We see from (7) and (32) that

$$
\begin{aligned}
\operatorname{ker} E & =\operatorname{ker}\left[\begin{array}{lll}
I & 0 & 0
\end{array}\right] V^{-1} \\
F^{-1} \operatorname{im} E & =\operatorname{ker}\left[\begin{array}{lll}
C_{0} & I & 0
\end{array}\right] V^{-1} \\
\operatorname{ker} G & =\operatorname{ker}\left[\begin{array}{lll}
G_{1} & G_{2} & G_{3}
\end{array}\right] V^{-1}
\end{aligned}
$$

Hence, we obtain

$$
(\mathrm{A} 5) \Leftrightarrow \operatorname{rank}\left[\begin{array}{ccc}
I & 0 & 0 \\
C_{0} & I & 0 \\
G_{1} & G_{2} & G_{3}
\end{array}\right] V^{-1}=n
$$

(full column rank)

$\Leftrightarrow G_{3}$ has full column rank.

$\Leftrightarrow R=G_{3}^{T} G_{3}>0$

It is also immediate from (7) and (32) that

$$
\begin{aligned}
(\mathrm{A} 4) \Leftrightarrow \operatorname{rank} & {\left[\begin{array}{ccc}
A_{0}-j \omega I & B_{0} & B \\
C_{0} & I & 0 \\
\hdashline G_{1} & G_{2} & G_{3}
\end{array}\right]=n, \forall \omega \in \mathbf{R} } \\
\Leftrightarrow & \operatorname{rank}\left[\begin{array}{cc}
A-j \omega I & B \\
C & D
\end{array}\right]=r+n-m, \forall \omega \in \mathbf{R} \\
& \text { (full column rank) }
\end{aligned}
$$

Since $(A, B)$ is controllable, the following ARE has a positive semi-definite stabilizing solution $P^{+}$if and only if $R>0$ and (34) hold ${ }^{18)}$.

$$
A^{T} P+P A-(P B+S) R^{-1}(P B+S)^{T}+Q=0
$$

Then, the optimal control is given by the state feedback

$$
u(t)=-K x(t), \quad K=R^{-1}\left(B^{T} P^{+}+S^{T}\right)
$$

and the closed-loop state-space system

$$
\dot{x}(t)=\left[A-B R^{-1}\left(B^{T} P^{+}+S^{T}\right)\right] x(t)
$$


is asymptotically stable. Hence, from (13) and (36), we see that the optimal interconnected system is stable, namely $\xi \in \mathcal{B}_{\text {sm }}\left(\xi_{0}\right)$ for all initial conditions $\xi_{0} \in \mathbf{R}^{n}$ and

$$
\xi(t)=V\left[\begin{array}{c}
I \\
-C_{0} \\
-K
\end{array}\right] x(t) \longrightarrow 0 \quad(t \rightarrow \infty)
$$

It is well known ${ }^{15)}$ that $P^{+}$is the maximal element of the set $\mathcal{P}=\left\{P \in \mathbf{R}^{r \times r}: L_{S S}(P) \geq 0\right\}$ and

$$
L_{S S}\left(P^{+}\right)=\left[\begin{array}{c}
K^{T} \\
I
\end{array}\right] R\left[\begin{array}{ll}
K & I
\end{array}\right] \geq 0
$$

where

$$
L_{S S}(P):=\left[\begin{array}{cc}
A^{T} P+P A & P B \\
B^{T} P & 0
\end{array}\right]+\left[\begin{array}{cc}
Q & S \\
S^{T} & R
\end{array}\right]
$$

We define $\left[\begin{array}{lll}H_{1} & H_{2} & H_{3}\end{array}\right]=H V$. Since $X^{+}$has the form of

$$
X^{+}=U^{T}\left[\begin{array}{ccc}
P^{+} & 0 & 0 \\
X_{4} & X_{5} & X_{6}
\end{array}\right] V^{-1}
$$

as shown in the proof of Theorem 2 (ii), we obtain

$$
\begin{aligned}
& L_{S S}\left(P^{+}\right)=N^{T} L\left(X^{+}\right) N=N^{T} H^{T} H N \\
& \quad=\left[\begin{array}{c}
\left(H_{1}-H_{2} C_{0}\right)^{T} \\
H_{3}^{T}
\end{array}\right]\left[\begin{array}{ll}
H_{1}-H_{2} C_{0} & H_{3}
\end{array}\right](41)
\end{aligned}
$$

where

$$
N:=V\left[\begin{array}{cc}
I & 0 \\
-C_{0} & 0 \\
0 & I
\end{array}\right]
$$

By comparing (41) with (39), we can without loss of generality write $H_{3}$ and $H_{1}-H_{2} C_{0}$ as

$$
H_{3}=R^{1 / 2}, \quad H_{1}-H_{2} C_{0}=R^{1 / 2} K
$$

Since $R$ is positive definite, we get

$$
\operatorname{rank} L\left(X^{+}\right)=\operatorname{rank} H=\operatorname{rank} R=n-m
$$

Moreover, we see from (8),(10) and (42) that

$$
\begin{aligned}
H \xi(t)=0 & \Leftrightarrow H_{1} x(t)+H_{2} f(t)+H_{3} u(t)=0 \\
& \Leftrightarrow\left(H_{1}-H_{2} C_{0}\right) x(t)+H_{3} u(t)=0 \\
& \Leftrightarrow R^{1 / 2} K x(t)+R^{1 / 2} u(t)=0 \\
& \Leftrightarrow u(t)+K x(t)=0
\end{aligned}
$$

This implies that the optimal control is given by (31).

The basic idea of the proof of Theorem 3 is to show that, under the assumption (A1)-(A3), the ARE (35) has a stabilizing solution if and only if (A4) and (A5) hold. These conditions guarantee the regularity of the LQ control problem for linear implicit system (see the reference 2) for exact definition of the regularity).
Although we have used the state-space results in the proofs of Theorems 2 and 3, we do not have to transform the linear implicit representation into the state-space one in order to solve the CLMI (15): In fact, the CLMI can be equivalently reduced to an LMI condition with the coefficient matrices of the original system (1).

\section{Alternative LMI Condition}

In this section, we will show that we have no numerical difficulty to solve the CLMI (15) by deriving an equivalent LMI condition without any equality constraints. This LMI condition is a generalization of the generalized Lyapunov inequality proposed for descriptor systems ${ }^{14)}$.

Theorem 4. If the CLMI (15) has a solution $X \in$ $\mathbf{R}^{m \times n}$, then it is expressed by

$$
X=\Pi E+W \Gamma
$$

where the pair $(\Pi, \Gamma) \in \mathbf{R}^{m \times m} \times \mathbf{R}^{(m-r) \times n}$ is a solution to the LMI:

$$
F^{T}(\Pi E+W \Gamma)+(\Pi E+W \Gamma)^{T} F+M \geq 0, \Pi=\Pi^{T}(44)
$$

and $W \in \mathbf{R}^{m \times(m-r)}$ is a constant matrix of full column rank such that ker $E^{T}=\operatorname{im} W$.

Conversely, if the LMI (44) has a solution $(\Pi, \Gamma)$, then $X:=\Pi E+W \Gamma$ satisfies the CLMI (15).

Proof. We first assume that (15) has a solution $X$. It is easy to verify that there exists a symmetric matrix $\Pi$ satisfying $E^{T} X=E^{T} \Pi E$ if $E^{T} X=X^{T} E$ holds. Then, we have $E^{T}(X-\Pi E)=0$, namely, $X-\Pi E \in \operatorname{ker} E^{T}$. Hence, $X=\Pi E+W \Gamma$ holds for some matrix $\Gamma$. Substituting $X=\Pi E+W \Gamma$ into (15b) yields (44).

Conversely, we assume that there exist $\Pi$ and $\Gamma$ satisfying (44). Then, by defining $X=\Pi E+W \Gamma$, we obtain (15b). Moreover, $E^{T} X=X^{T} E=E^{T} \Pi E$ immediately follows from $E^{T} W=0$.

\section{Conclusion}

In this paper, we have considered the dissipativity and the LQ optimal control for linear implicit systems. We have derived a new necessary and sufficient condition for the dissipativity in terms of a CLMI. Based on this CLMI condition, we have shown that the optimal control law is given as an algebraic constraint among system variables in the case where the weighting matrix is positive semidefinite. The author believes that the present results on the dissipativity will be helpful for further study of linear implicit systems (robust control, stability analysis and so on). 


\section{References}

1) J.D. Aplevich: Implicit Linear Systems, Springer-Verlag (1991)

2) T. Geerts: Regularity and singularity in linear-quadratic control subject to implicit continuous-time systems, Circuits Syst. Signal Process., 13-1, 19/30 (1994)

3) T. Geerts: Linear-quadratic control with and without stability subject to general implicit continuous-time systems: Coordinate-free interpretations of the optimal costs in terms of dissipation inequality and linear matrix inequality; Existence and uniqueness of optimal controls and state trajectories, Linear Algebra and its Applications, 203204, 607/658 (1994)

4) A.H.W. Geerts and J.M. Schumacher: Impulsive-smooth behavior in multimode systems Part I: State-space and polynomial representations, Automatica, 32-5, 747/758 (1996)

5) A.H.W. Geerts and J.M. Schumacher: Impulsive-smooth behavior in multimode systems Part I: Minimality and equivalence, Automatica, 32-6, 819/832 (1996)

6) R. van der Geest and A. Rantzer: Linear quadratic control under quadratic constraints, Proc. 4th European Contr. Conf., TH-A-D3 (1997)

7) M.L.J. Hautus and L.M. Silverman: System structure and singular control, Linear Algebra and its Applications, 50, $369 / 402$ (1983)

8) A. Kawamoto and T. Katayama: The dissipation inequality and generalized algebraic Riccati equation for linear quadratic control problem of descriptor system, Trans. Inst. Syst. Contr. \& Inform. Eng., 10-8, 412/421 (1997) (in Japanese); also Proc. of the 13th IFAC World Congress, D, 103/108 (1996)

9) M. Kuijper: First-order Representations of Linear Systems, Birkhäuser (1994)

10) I. Masubuchi and E. Shimemura: Stability analysis of implicit systems with possible impulses, Trans. Soc. Instr. Conr. Eng., 35-1, 66/70 (1999) (in Japanese).; also An LMI condition for stability of implicit systems, Proc. 36th IEEE Conf. Decision \& Contr., 779/800 (1997)

11) T. Shiotsuki, K. Shbasato and T. Sato: A behavioral approach by using pencil based models, Proc. 11th IFAC Symposium on System Identification (SYSID 'gr), $1231 / 1236$ (1997)

12) K. Takaba, N. Morihira and T. Katayama: A generalized Lyapunov theorem for descriptor systems, Syst. Es Contr. Letters, 24-2, 49/51 (1995)

13) H.L. Tentelman and J.C. Willems: Every storage function is a state function, Syst. \& Contr. Lett. (1997)

14) E. Uezato and M. Ikeda: A strict LMI condition for stability of linear descriptor systems and its application to robust stabilization, Trans. Soc. Instr. Meas. Contr. Eng., 34-12, 1854/1860 (1998) (in Japanese)

15) J.C Willems: Least squares stationary optimal control and the algebraic Riccati equation, IEEE Trans. Automat. Contr., 16, 621/634 (1971)

16) J.C. Willems: Paradigms and puzzles in the theory of dynamical systems, IEEE Trans. Automat. Contr., 36-3, 259/294 (1991)

17) J.C. Willems and H.L. Trentelman: On quadratic differential forms, SIAM J. Contr. \&\& Optim., 36-5, 1703/1749 (1998)

18) K. Zhou, J. Doyle and K. Glover: Robust and Optimal Control, Prentice-Hall (1996)

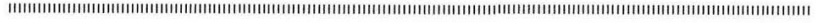

Kiyotsugu Такава (Member)

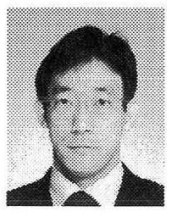

Kiyotsugu Takaba received B.Eng. degree in applied mathematics and physics, M.Eng. degree in applied systems science and Dr.Eng. degree in applied mathematics all from Kyoto University in 1989, 1991 and 1996, respectively. Since 1991, he has been with the Department of Applied Mathematics and Physics in Kyoto University, where he is presently an Associate Professor. His research interest includes robust and optimal control for multivariable dynamical systems. He is a member of IEEE, ISCIE and SICE.

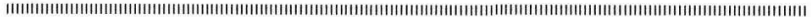

\section{Appendix A. Impulsive-Smooth Distribution}

We here give the definition of impulsive-smooth distributions. For more detail on their properties, the readers should refer to2),3),7) and the references therein.

We denote the set of distributions defined on $\mathbf{R}$ with support on $[0, \infty)$ by $\mathcal{K}$. This set is closed under convolution. It should be noted that typical example of the elements of $\mathcal{K}$ is the Dirac delta distribution $\delta$ and its derivatives.

Linear combinations of the delta distribution and its derivatives are called impulsive distributions, and the set of these distributions is denoted by $\mathcal{C}_{\text {p-imp. }}$ A general form of an impulsive distribution is given by $\sum_{i=0}^{\ell} c_{i} \delta^{(i)}$, where $c_{i}(i=0, \cdots, \ell)$ is a real-valued constant vector with appropriate dimension, and where $\delta^{(i)}$ denotes the $i$-th derivative of $\delta$.

Another important subset of $\mathcal{K}$ is the set of smooth distributions $\mathcal{C}_{\mathrm{sm}}$, which can be identified with the functions continuously differentiable on the interval $(0, \infty)$.

We define the set of impulsive-smooth distributions as the direct sum of $\mathcal{C}_{\mathrm{p} \text {-imp }}$ and $\mathcal{C}_{\mathrm{sm}}$ :

$$
\mathcal{C}_{\text {imp }}=\mathcal{C}_{\mathrm{p} \text {-imp }}+\mathcal{C}_{\mathrm{sm}} \subset \mathcal{K}
$$

The element $x \in \mathcal{C}_{\mathrm{imp}}$ is expressed as

$$
x=x_{\mathrm{p}-\mathrm{imp}}+x_{\mathrm{sm}}, \quad x_{\mathrm{p}-\mathrm{imp}} \in \mathcal{C}_{\mathrm{p}-\mathrm{imp}}, x_{\mathrm{sm}} \in \mathcal{C}_{\mathrm{sm}}
$$

\section{Appendix B. Dissipative State-Space System}

We consider a linear state-space system described by

$$
\dot{x}(t)=A x(t)+B u(t), \quad x(0-)=x_{0}
$$

where $x(t) \in \mathbf{R}^{n}$ and $u(t) \in \mathbf{R}^{m}$ are the state vector and the input vector, respectively.

Definition B.1. The state-space system (B. 1) is said to be dissipative with respect to $s(x, u)$ if there exists a 
function $v(x)$ satisfying $v(0)=0$ and the dissipation inequality:

$$
\begin{array}{r}
\int_{0-}^{\tau} s(x(t), u(t)) d t+v(x(\tau)) \geq v\left(x_{0}\right) \\
\forall \tau \geq 0, \forall x_{0} \in \mathbf{R}^{n}
\end{array}
$$

The functions $s(x, u)$ and $-v(x)$ are called a supply rate and storage function, respectively.

The following results are due to Willems ${ }^{15}$ ).

Lemma B.1. Assume that the system (B. 1) is controllable. Then, the following statements are equivalent.

(i) The system (B.1) is dissipative with respect to $s(x, u)$.

(ii) $v^{+}\left(x_{0}\right)>-\infty, \forall x_{0} \in \mathbf{R}^{n}$

(iii) $\int_{0-}^{T} s(x, u) d t \geq 0$ holds for any $T \geq 0$ and for any $(x, u)$ satisfying $x(0-)=x(T)=0$.

The function $v^{+}$is defined by

$$
v^{+}\left(x_{0}\right)=\inf _{u \in \mathcal{C}_{\text {sin }}}\left\{\int_{0-}^{\infty} s(x(t), u(t)) d t \mid \lim _{t \rightarrow \infty} x(t)=0\right\}
$$

Moreover,

$$
v(x) \leq v^{+}(x), \quad \forall x \in \mathbf{R}^{n}
$$

holds for every storage function $v(x)$.

Lemma B.2. Suppose that the quadratic supply rate is given by

$$
s(x, u)=\left[\begin{array}{l}
x \\
u
\end{array}\right]^{T}\left[\begin{array}{cc}
Q & S \\
S^{T} & R
\end{array}\right]\left[\begin{array}{l}
x \\
u
\end{array}\right]
$$

Then, the following statements are equivalent.

(i) The system (B. 1) is dissipative with respect to the supply rate $s(x, u)$.

(ii) There exists a symmetric matrix $P$ satisfying the following LMI.

$$
\left[\begin{array}{cc}
A^{T} P+P A & P B \\
B^{T} P & 0
\end{array}\right]+\left[\begin{array}{cc}
Q & S \\
S^{T} & R
\end{array}\right] \geq 0
$$

If such a $P$ exists, $v(x):=x^{T} P x$ satisfies the dissipation inequality (B. 2). In this case, we let $\mathcal{P}$ be the set of all solutions of (B. 3). Then there exists a $P^{+} \in \mathcal{P}$ such that

$$
P \leq P^{+}, \forall P \in \mathcal{P}
$$

Moreover, we have

$$
v^{+}(x)=x^{T} P^{+} x
$$

